\title{
Morse cohomology in a Hilbert space via the Conley index
}

\author{
Maciej Starostka
}

\begin{abstract}
The main theorem of this paper states that Morse cohomology groups in a Hilbert space are isomorphic to the cohomological Conley index. It is also shown that calculating the cohomological Conley index does not require finite-dimensional approximations of the vector field. Further directions are discussed.
\end{abstract}

Mathematics Subject Classification. Primary 53D40; Secondary 55N20.

Keywords. Morse homology, Morse-Witten-Floer complex, Conley index, Seiberg-Witten-Floer homology.

\section{Introduction}

The aim of this paper is to show that Morse cohomology groups defined for a certain functional in a Hilbert space can be recovered via the Conley index. This was motivated by the growing number of different Floer cohomology theories in three- and four-dimensional topology. A lot of applications come from the fact that some of those theories are equivalent. As an example, let us mention the Seiberg-Witten-Floer cohomology and the embedded contact Floer cohomology. The equivalence between those two was used to find Reeb orbits on contact manifolds. Some stronger versions of the Weinstein conjectures were obtained (cf. [Tau]).

However, some of the Floer theories are still conjectured to be equivalent, e.g. Seiberg-Witten-Floer (HSW) cohomology and Monopole-Floer cohomology (HM). The former is defined by the Conley index while the latter one by counting connecting orbits. The idea of using the Conley index instead of Floer theory for Seiberg-Witten equations was first introduced by Manolescu in [Man]. One of the motivations was the fact that we do not have to deal with transversality.

Our approach to the Floer theory via the Conley index is slightly different from that in [Man]. We would like to work with an index pair in a Hilbert space and apply the concept of Gęba-Granas cohomology (see [G-G]). This 
allows us to avoid finite-dimensional approximations of the vector field. Results presented below are obtained by the facts that those cohomology groups satisfy axioms of the generalized cohomology theory (see below for a precise statement) and that they are invariant under the flow deformations. Those two facts were proved by Abbondandolo in [Abb97].

\section{E-cohomology}

E-cohomology groups are defined to be the direct limit of certain ordinary cohomology groups. Let us remark on the latter first. The main requirement is that the theory satisfies the strong excision axiom. There are various possible choices, e.g. Cech cohomology groups [G-G] or Alexander-Spanier cohomology groups [Abb97]. We choose the homotopical point of view. Let us recall the definition from the Appendix in [C-J] (cf. [AGP, Chap. 7]). Denote by $K(\mathbb{F}, n)$ an appropriate Eilenberg-Maclane space. For a topological pair $(X, Y)$ define cohomology groups by

$$
H^{n}(X, Y)=[X \cup \mathrm{CY}, K(\mathbb{F}, n)],
$$

where CY is a cone on $Y$. In the case when $X$ is a compact Hausdorff space and $Y$ is its closed subset, $H^{n}(X, Y)$ coincides with the Alexander-Spanier cohomology group and

$$
H^{n}(X, Y)=[X / Y, K(\mathbb{F}, n)] .
$$

One can also define cohomology groups with compact supports of a locally compact Hausdorff space $U$ by

$$
H_{c}^{n}(U)=H^{n}\left(U^{+}, *\right)=[U, K(\mathbb{F}, n)]_{c},
$$

where $U^{+}$denotes the one-point compactification of $U$ and $[,]_{c}$ denotes homotopy classes of compactly supported maps.

Throughout the rest of the paper, we take $\mathbb{F}=\mathbb{Z}_{2}$.

We are now ready to give an overview on what $E$-cohomology is. Let $E$ be a Hilbert space with a splitting $E=E^{+} \oplus E^{-}$, where each of $E^{+}$and $E^{-}$ is either infinite dimensional or trivial. We say that $\left\{E_{n}\right\}_{n \in \mathbb{N}}$ is an approximating system for $E$ if

(1) $E_{n}$ is a finite-dimensional subspace of $E$ for every $n$;

(2) there is an inclusion $i_{n, n^{\prime}}: E_{n} \hookrightarrow E_{n^{\prime}}$ for every $n^{\prime}>n$;

(3) $\overline{\bigcup_{n} E_{n}}=E$.

We recall the definition of $E$-cohomology in two extremal cases: (i) when $E^{+}=\{0\}, E^{-}=l^{2}$ and (ii) when $E^{+}=l^{2}, E^{-}=\{0\}$. For $l^{2}$ we take an approximation system induced by the spaces of finite sequences. However, one can prove (see [G-G], [Abb97]) that the definition does not depend on the choice of the approximation system.

Let us first consider the case of $E=\{0\} \oplus l^{2}$. Take a closed and bounded set $X \subset E$. We define the finite-codimensional cohomology in the following 
way (see $[\mathrm{G}-\mathrm{D}])$. Put

$$
\begin{aligned}
& E_{n}=\left\{\left(x_{1}, x_{2}, \ldots\right) \in E: x_{k}=0 \text { for } k>n\right\}, \\
& \hat{E}_{n}=\left\{\left(x_{1}, x_{2}, \ldots\right) \in E_{n}: x_{n} \geq 0\right\}, \\
& \check{E}_{n}=\left\{\left(x_{1}, x_{2}, \ldots\right) \in E_{n}: x_{n} \leq 0\right\}, \\
& X_{n}=X \cap E_{n}, \quad \hat{X}_{n}=X \cap \hat{E}_{n}, \quad \check{X}_{n}=X \cap \check{E}_{n} .
\end{aligned}
$$

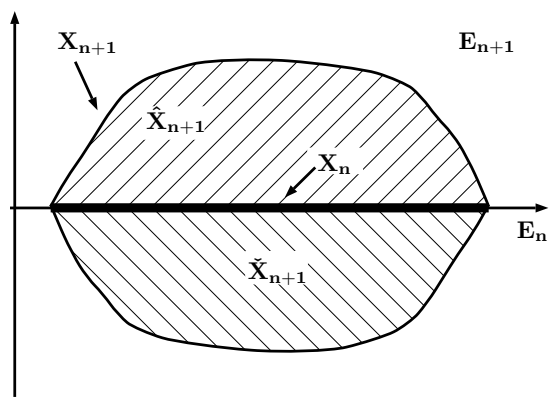

FiguRE 1

Since $\hat{\mathrm{X}}_{n+1} \cap \check{\mathrm{X}}_{n+1}=X_{n}$ and $\hat{\mathrm{X}}_{n+1} \cup \check{\mathrm{X}}_{n+1}=X_{n+1}$, the Mayer-Vietoris sequence for a triad $\left(X_{n+1}, \hat{\mathrm{X}}_{n+1}, \check{\mathrm{X}}_{n+1}\right)$ gives a homomorphism

$$
\delta_{n}: H^{k}\left(X_{n}\right) \rightarrow H^{k+1}\left(X_{n+1}\right) .
$$

Definition 2.1. Finite-codimensional cohomology groups on $l^{2}$ are defined by

$$
H_{E}^{k}(X)=\lim _{\longrightarrow}\left(H^{\operatorname{dim} E_{n}+k}\left(X_{n}\right), \delta_{n}\right) .
$$

Notice that if $k>0$, then $\operatorname{dim} E_{n}+k>\operatorname{dim} X_{n}$. Thus, the above groups can be nontrivial only for negative $k$. We have chosen a convention which is compatible with the one in [Abb97] and opposite to that in [G-D]. This would be more convenient when we deal with the case when both $E^{+}$and $E^{-}$are nonzero.

As a simplest nontrivial example, take $X=S(E)$; i.e., $X$ is a unit sphere in $E$. Then $X_{n}=S\left(E_{n}\right), H^{n-1}\left(X_{n}\right)=\mathbb{Z}_{2}$ and all the maps $\delta_{n}$ are isomorphisms. Thus

$$
H_{E}^{-1}(S(E)) \simeq \mathbb{Z}_{2}
$$

and $H_{E}^{k}(S(E))$ is trivial if $k \neq-1$ (notice that since $H^{0}\left(S\left(E_{n}\right)\right.$ ) is mapped by $\delta_{n}$ to $H^{\operatorname{dim} E_{n+1}-\operatorname{dim} E_{n}}\left(S\left(E_{n+1}\right)\right)$, we do not see zeroth cohomology of the sphere). Let us also emphasize that if $X$ is compact (in particular, if it is contained in a finite-dimensional subspace), then all the $E$-cohomology groups are trivial. For a general separable Hilbert space $E=\{0\} \oplus E^{-}$we can take an isomorphism with $l^{2}$ (i.e., choose an approximating system) and repeat the construction. 
This simple concept can be generalized in many directions. Gȩba and Granas [G-G] proved that for any generalized cohomology theory, the above groups are well defined. For example, taking cohomotopy groups instead of cohomology groups gives us stable cohomotopy groups. In addition, they proved that the resulting theory is always a generalized cohomology theory on the Leray-Schauder category. Morphisms of the Leray-Schauder category are compact fields (i.e., Id $+K$ where $K$ is compact) so one could try to apply the above techniques to fixed point theory.

However, it is good to extend the set of morphisms of the LeraySchauder category. One reason for this is that one cannot compare spheres of different radii via maps $\mathrm{Id}+K$. Obviously, such spheres should have the same cohomology groups.

First of all, notice that the above cohomology groups are trivially invariant under translations. Abbondandolo proved that they are also invariant under the flow deformations. This allows us to compare spheres of different radii and also, which is more important, to use Morse theory and the Conley index techniques.

Another feature of [Abb97], [K-Sz] and [Sz] is a generalization to the socalled middle-dimensional cohomology, i.e., the case when both $E^{+}$and $E^{-}$ are of infinite dimension. Before introducing that, let us consider a second extremal example: $E^{+}=l^{2}, E^{-}=\{0\}$. In this case, $E$-cohomology groups are defined by

$$
H_{E}^{k}(X)=\lim _{\longrightarrow}\left(H^{k}\left(X_{m}, \delta_{m}^{\prime}\right)\right),
$$

where $\delta_{m}^{\prime}$ is induced by the inclusion of $X_{m}$ into $X_{m+1}$. One can easily see that if $X$ is a sphere in $E_{m}$, then $H_{E}^{k}(X)$ is nontrivial (and equal to $\mathbb{Z}_{2}$ ) only if $k=m-1$ or $k=0$. In fact, it is true that if $X$ is locally compact, then $H_{E}^{*}$ is isomorphic to the compactly supported cohomology mentioned above.

Now the middle-dimensional cohomology groups are defined by

$$
H_{E}^{k}(X)=\lim _{\longrightarrow}\left(H^{\operatorname{dim} E_{n}^{-}+k}\left(X_{(m, n)}, \delta_{m}^{\prime}, \delta_{n}\right)\right),
$$

where $X_{m, n}=X \cap\left(E_{m} \oplus E_{n}\right), \delta_{n}: X_{m, n} \rightarrow X_{m, n+1}$ is the map from the Mayer-Vietoris sequence and $\delta_{m}^{\prime}: X_{m, n} \rightarrow X_{m+1, n}$ is the map induced by inclusion. Again, this definition does not depend on the approximating system.

One can think of $E$-cohomology as cohomology of finite-codimensional cohomology with respect to $E^{-}$and cohomology with compact supports with respect to $E^{+}$.

We would like to emphasize the fact that $E$-cohomology groups satisfy axioms of a generalized cohomology theory (see [Abb97, Theorem 0.2]). All the results presented below can be obtained by these axioms without the knowledge of the precise construction of $E$-cohomology groups. For the sake of completeness, let us recall the homotopy invariance, the strong excision axiom and the long exact sequence for a triple.

Definition 2.2. A continuous map $\Psi:(X, A) \rightarrow(Y, B)$ is an E-morphism if

(1) it has the form

$$
\Psi(x)=L x+K(x)
$$


where $L$ is a linear automorphism of $E$ such that $L E^{+}=E^{+}$and $K$ maps bounded sets into precompact sets;

(2) $\Psi^{-1}(U)$ is bounded for every bounded set $U$.

We also say that $E$-morphisms $\Phi$ and $\Phi^{\prime}$ from $(X, A)$ to $(Y, B)$ are $E$ homotopic if there exists an $E$-homotopy joining them, i.e., a continuous map $\Psi:(X, A) \times[0,1] \rightarrow(Y, B)$ such that

(1) $\Psi(x, t)=L_{t} x+K(x, t)$, where $L_{t}$ is a linear automorphism of $E$ and $K$ maps bounded sets into precompact sets;

(2) $\Psi^{-1}(U)$ is bounded for every bounded set $U$;

(3) $\Psi(\cdot, 0)=\Phi$ and $\Psi(\cdot, 1)=\Phi^{\prime}$.

The above definitions allow us to state the following.

- Homotopy invariance: if two $E$-morphisms $\Phi$ and $\Phi^{\prime}$ are $E$-homotopic, then $H_{E}^{*}(\Phi)=H_{E}^{*}\left(\Phi^{\prime}\right)$.

- Strong excision: if $X$ and $Y$ are closed and bounded subsets of $E$ and $i:(X, X \cap Y) \rightarrow(X \cup Y, Y)$ is the inclusion map, then $H_{E}^{*}(i)$ is an isomorphism.

- Long exact sequence: for a triple $X \subset Y \subset Z$ of closed and bounded sets we have a long exact sequence:

$$
\begin{aligned}
\cdots \rightarrow H_{E}^{k}(Z, Y) & \rightarrow H_{E}^{k}(Z, X) \rightarrow H_{E}^{k}(Y, X) \\
& \stackrel{\delta}{\rightarrow} H_{E}^{k+1}(Z, Y) \rightarrow H_{E}^{k+1}(Z, X) \rightarrow \cdots .
\end{aligned}
$$

In the proof of Proposition 3.4 we will also need the following two lemmas (cf. [Abb97, pp. 372-373]).

Lemma 2.3. Let $Y$ be a closed subset of $X$. If there exists an $E$-homotopy

$$
\Psi:(X, A) \times[0,1] \rightarrow(X, A)
$$

such that $\Psi_{0}=\mathrm{Id}, \Psi_{1}(X) \subset Y$ and $\Psi_{t}(Y) \subset Y$ for every $t \in[0,1]$, then

$$
H_{E}^{*}(X, A) \simeq H_{E}^{*}(Y, A),
$$

the isomorphism being induced by the inclusion map.

Lemma 2.4. Let $B$ be a closed subset of $A$. If there exists an $E$-homotopy

$$
\Psi:(X, A) \times[0,1] \rightarrow(X, A)
$$

such that $\Psi_{0}=\mathrm{Id}, \Psi_{1}(A) \subset B$ and $\Psi_{t}(B) \subset B$ for every $t \in[0,1]$, then

$$
H_{E}^{*}(X, A) \simeq H_{E}^{*}(X, B),
$$

the isomorphism being induced by the inclusion map.

\section{Conley index}

We make the following assumptions throughout this section.

Let $f \in C^{2}(E, \mathbb{R})$ be a function of the form

$$
f(x)=\frac{1}{2}\langle L x, x\rangle+b(x),
$$


where $L$ is a self-adjoint isomorphism, $\nabla b(x)$ is globally Lipschitz and $D^{2} b(x)$ is compact for every $x \in E$. Then the operator $L$ gives a splitting of $E$ into $E^{+}$and $E^{-}$corresponding to positive and negative eigenspaces, respectively. We would like to work with flows generated by the minus-gradient equations; i.e.,

$$
\dot{x}=-\nabla f(x) .
$$

We define the cohomological Conley index in a Hilbert space $E$ as the $E$-cohomology of an index pair in $E$. This is a different approach than in [Izy] and $[\mathrm{Man}]$, because it does not use finite-dimensional approximations of the vector field. We compare our approach to [Izy] after proving Proposition 3.4.

Following [GIP, p. 221], we define an isolating neighborhood in a Hilbert space.

Definition 3.1. We say that a bounded and closed set $N \subset E$ is an isolating neighborhood if

$$
\operatorname{Inv}(N) \subset \operatorname{int} N .
$$

Definition 3.2. Let $N$ be an isolating neighborhood of an invariant set $S$. We call a closed and bounded pair $\left(N_{1}, N_{0}\right)$ an index pair for $S$ if

(1) $N_{0}$ is positively invariant relative to $N_{1}$,

(2) $S \subset \operatorname{int} N_{1} \backslash N_{0}$,

(3) if $\gamma \in N_{1}, t>0$ and $\gamma \cdot t \notin N$, then there exists $t^{\prime}$ such that $\gamma \cdot\left[0, t^{\prime}\right] \subset N_{1}$ and $\gamma \cdot t^{\prime} \in N_{0}$.

Moreover, we say that an index pair is regular if the function

$$
\tau(x)=\inf \left\{s \in \mathbb{R}^{\geq 0}: x \cdot[0, s] \not \subset N_{1} \backslash N_{0}\right\}
$$

is continuous.

Unless otherwise stated, we assume that all index pairs are regular (cf. Remark 3.6 for the existence).

We say that an index pair $\left(N_{1}, N_{0}\right)$ is contained in the isolating neighborhood $N$ if $N_{0} \subset N_{1} \subset N$ and $N_{1}, N_{0}$ are positively invariant relative to $N$ (cf. [Smol, p. 489]).

Definition 3.3. We define the cohomological Conley index of $S$ (denoted by $\left.\operatorname{ch}^{*}(S)\right)$ to be $H_{E}^{*}\left(N_{1}, N_{0}\right)$, where $\left(N_{1}, N_{0}\right)$ is an index pair for $S$.

The above definition only makes sense if we prove the independence of a choice of index pairs. This is stated in the following proposition.

Proposition 3.4. Suppose that we have a flow as in the beginning of this section. Let $\left(N_{1}, N_{0}\right),\left(\hat{N}_{1}, \hat{N}_{0}\right)$ be two regular index pairs for $S=\operatorname{Inv}(N)$ 
contained in the same isolating neighborhood $N$. Then

$$
H_{E}^{*}\left(N_{1}, N_{0}\right) \simeq H_{E}^{*}\left(\hat{N}_{1}, \hat{N}_{0}\right) .
$$

Notice that the assumption on the flow is crucial.

Define the sets $N_{1}^{t}, N_{0}^{-t}$ by

$$
\begin{aligned}
N_{1}^{t} & =\left\{x \in N_{1}: x \cdot[-t, 0] \subset N_{1}\right\}, \\
N_{0}^{-t} & =\left\{x \in N_{1}: \exists_{y \in N_{0}} \exists_{t^{\prime} \in[0, t]} \text { s.t. } y \cdot\left[-t^{\prime}, 0\right] \subset N_{1}, y \cdot\left(-t^{\prime}\right)=x\right\} .
\end{aligned}
$$
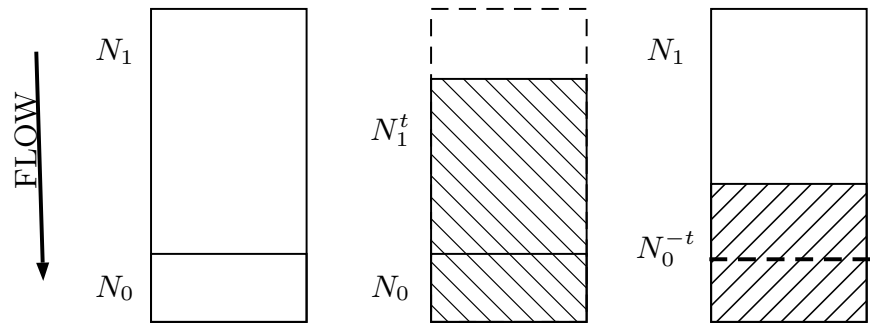

FiguRE 2

The proof of Proposition 3.4 can be divided into three steps.

Step 1 . For every $t>0$ there is an isomorphism

$$
H_{E}^{*}\left(N_{1}, N_{0}\right) \rightarrow H_{E}^{*}\left(N_{1}^{t}, N_{0} \cap N_{1}^{t}\right) .
$$

Step 2. For every $t>0$ there is an isomorphism

$$
H_{E}^{*}\left(N_{1}, N_{0}\right) \rightarrow H_{E}^{*}\left(N_{1}, N_{0}^{-t}\right) .
$$

Step 3. There exists $T>0$ such that

$$
\begin{aligned}
& \left(N_{1}^{T}, N_{0} \cap N_{1}^{T}\right) \subset\left(\hat{N}_{1}, \hat{N}_{0}^{-T}\right), \\
& \left(\hat{N}_{1}^{T}, \hat{N}_{0} \cap \hat{N}_{1}^{T}\right) \subset\left(N_{1}, N_{0}^{-T}\right)
\end{aligned}
$$

and the inclusions induce isomorphisms of $E$-cohomology groups.

Proof.

Step 1. Define $\Psi:\left(N_{1}, N_{0}\right) \times[0,1] \rightarrow\left(N_{1}, N_{0}\right)$ by

$$
\Psi(x, s)= \begin{cases}x \cdot s & \text { if } x \cdot[0, s] \in N_{1} \backslash N_{0} \\ x \cdot \tau(x) & \text { otherwise. }\end{cases}
$$

Put $X=N_{1}, Y=N_{1}^{t} \cup N_{0}, A=N_{0}$. Lemma 2.3 gives us

$$
H_{E}^{*}\left(N_{1}, N_{0}\right) \simeq H_{E}^{*}\left(N_{1}^{t} \cup N_{0}, N_{0}\right) \text {. }
$$

From the excision axiom we have

$$
H_{E}^{*}\left(N_{1}^{t}, N_{0} \cap N_{1}^{t}\right) \simeq H_{E}^{*}\left(N_{1}^{t} \cup N_{0}, N_{0}\right) .
$$


Step 2. This can be done in a similar way as Step 1.

Step 3. Take $\hat{N}=\operatorname{cl}\left(N \backslash N_{1}\right)$. For every $x \in \hat{N}$ there exists $T_{x}>0$ such that $x \cdot\left(-T_{x}\right) \notin \hat{N}$. In fact, we will show that there exists $T_{1}$ which satisfies the above condition for every $x \in \hat{N}$. In a finite-dimensional case this is just a consequence of the compactness of $\hat{N}$.

Suppose we have a sequence $\left(y_{n}\right) \subset \hat{N}$ such that $y_{n} \cdot(-2 n, 0) \subset \hat{N}$. Put $x_{n}:=y_{n} \cdot(-n)$. Then both sets $x_{n} \cdot(-n, 0)$ and $x_{n} \cdot(0, n)$ are contained in $\hat{N}$. If $x_{n_{k}} \rightarrow x_{0}$, then $x_{0} \cdot(-\infty, 0) \subset \hat{N}$ and $x_{0} \in \hat{N}$ and we have arrived at a contradiction.

We now prove that if $x_{n} \cdot(-n, n) \subset \hat{N}$ for every $n$, then $\left(x_{n}\right)$ contains a convergent subsequence.

Suppose that $\left(x_{n}^{+}\right) \subset E^{+}$does not have a convergent subsequence. Then there exists $\epsilon>0$ such that $\left|x_{n}^{+}-x_{m}^{+}\right|>\epsilon$ for every $n \neq m$.

Take $s, T_{1}>0$ such that

$$
N \subset B(0, s), \quad\left|e^{T_{1} L} x\right|>\frac{3 s}{\epsilon}|x|
$$

for every $x \in E^{+}$. Then for $n, m>T_{1}$ we have

$$
\begin{aligned}
3 s & <\left|e^{T_{1} L}\left(x_{n}-x_{m}\right)\right| \\
& \leqslant\left|x_{m} \cdot T_{1}\right|+\left|x_{n} \cdot T_{1}\right|+\left|K\left(x_{m}, T_{1}\right)-K\left(x_{n}, T_{1}\right)\right| \\
& \leqslant 2 s+\left|K\left(x_{m}, T_{1}\right)-K\left(x_{n}, T_{1}\right)\right|
\end{aligned}
$$

and so

$$
\left|K\left(x_{m}, T_{1}\right)-K\left(x_{n}, T_{1}\right)\right|>s
$$

for every $n, m>T_{1}$. However, $K\left(\cdot, T_{1}\right)$ is compact and we have a contradiction. As a consequence, we can choose a convergent subsequence $\left(x_{n_{l}}^{+}\right)$. In a similar way, from $\left(x_{n_{l}}^{-}\right)$we can take a convergent subsequence $\left(x_{n_{k}}^{-}\right)$and this gives us a convergence of $\left(x_{n_{k}}\right)$.

By the same argument we can find $T_{2}>0$ such that for every $x \in N_{0}$ we have $x \cdot T \notin N_{0}$. Take

$$
T=\max \left\{T_{1}, T_{2}, \overline{T_{1}}, \overline{T_{2}}\right\},
$$

where $\overline{T_{1}}, \overline{T_{2}}$ correspond to the pair $\left(\hat{N}_{1}, \hat{N}_{0}\right)$. Then

$$
\begin{aligned}
& \left(N_{1}^{T}, N_{0} \cap N_{1}^{T}\right) \subset\left(\hat{N}_{1}, \hat{N}_{0}^{-T}\right), \\
& \left(\hat{N}_{1}^{T}, \hat{N}_{0} \cap \hat{N}_{1}^{T}\right) \subset\left(N_{1}, N_{0}^{-T}\right) .
\end{aligned}
$$

The proof that the above inclusions induce isomorphisms on the cohomology groups runs as in the finite-dimensional case (see [Smol, pp. 486-492] or [Ch, p. 401]).

Remark 3.5. One can show the independence of the Conley index without the assumption that both index pairs are contained in the same isolating neighborhood (cf. [Smol, p. 491]). 
Remark 3.6. We want to emphasize that for an isolated invariant set $S$ there exists a regular index pair. Let $U$ be an isolating neighborhood and define

$$
G^{T}(U)=\bigcap_{|t| \leqslant T} U \cdot(-t, t) .
$$

Then there exists $T>0$ such that $G^{T}(U) \subset \operatorname{int} U$.

Suppose the converse; i.e.,

$$
G^{n}(U) \not \subset \operatorname{int} U \text { for every } n \text {. }
$$

Take $x_{n} \in G^{n}(U) \backslash \operatorname{int} U$; i.e., $x_{n} \cdot(-n . n) \subset U$. There exists a convergent subsequence $x_{n_{k}} \rightarrow x_{0} \in S \subset \operatorname{int} U$ (see the proof above); a contradiction.

This proves that $G^{T}(U) \subset \operatorname{int} U$ for some $T>0$. For such a $U$ one can construct a regular index pair (see [Ch, Theorem 5.5.13]).

Let us compare our definition of the cohomological Conley index to the one which uses finite-dimensional approximations of the vector field [Izy]. For a compact $K$ define $K_{n}: E \rightarrow E$ by

$$
K(x)=P_{n} \circ K \circ P_{n}(x) .
$$

Let $S$ be an isolated invariant set for the flow generated by $F=L+K$ and let $\hat{N}$ be an isolating neighborhood for $S$. Then $F$ is related by continuation (through $E$-homotopies) to $F_{n_{0}}=L+K_{n_{0}}$ for sufficiently large $n_{0}$. Let $(N, L)$ be an index pair for the approximation, i.e., for the finite-dimensional flow generated by $F_{n_{0} \mid E_{n_{0}}}: E_{n_{0}} \rightarrow E_{n_{0}}$. Clearly

$$
\left(N_{E}, L_{E}\right)=\left(N \times D_{-n}^{+} \times D_{-n}^{-}, L \times D_{-n}^{+} \times D_{-n}^{-} \cup N \times D_{-n}^{+} \times \partial D_{-n}^{-}\right)
$$

is an index pair for $F$ in $E$, where $D_{-n}^{+/-}$denotes a disc in $E_{n}^{\perp} \cap E^{+/-}$. It is easy to check that $H_{E}^{*}\left(N_{E}, L_{E}\right)$ coincides with the cohomological Conley index defined in $[\mathrm{Izy}]$. Since $E$-cohomology does not depend on the index pair, those two approaches coincide in general.

\section{Main theorem}

Let us recall that we are interested in a flow generated by the minus gradient vector field for a function $f \in C^{2}(E, \mathbb{R})$ of the form

$$
f(x)=\frac{1}{2}\langle L x, x\rangle+b(x),
$$

where $L$ is a self-adjoint isomorphism, $\nabla b(x)$ is globally Lipschitz and $D^{2} b(x)$ is compact for every $x \in E$.

Let $S$ be a compact isolated invariant set containing only nondegenerate critical points $x_{1}, \ldots, x_{n}$ and orbits connecting them.

For a nondegenerate critical point $x$ we define an $E$-index by

$$
\operatorname{ind}_{E} x=\operatorname{dim} V \cap E^{+}-\operatorname{dim} V^{\perp} \cap E^{-}=\operatorname{dim} V \cap E^{+}-\operatorname{codim} E^{+}+V,
$$

where $V$ is the negative eigenspace of $D^{2} f(x), E^{+}$and $E^{-}$are, respectively, positive and negative eigenspaces of $L$. 
Suppose further that the transversality condition holds; i.e., if

$$
\operatorname{ind}_{E} y-\operatorname{ind}_{E} x=1,
$$

the stable manifold of $y$ and unstable of $x$ intersect transversally.

Main Theorem. We have

$$
H F^{*}(S) \simeq \operatorname{ch}^{*}(S),
$$

where $H F^{*}(S)$ denotes Floer cohomology.

Salamon [Sal] used an analogous theorem in the following way. Take a function $f$ on finite-dimensional closed manifold $M$. Then $(M, \emptyset)$ is an index pair for the isolated invariant set $S=M$. If the Morse cohomology is isomorphic to the cohomological Conley index, we have

$$
H^{*}(M)=H^{*}(M, \emptyset) \simeq H_{\text {Morse }}^{*}(M) .
$$

Thus, this is just another proof that Morse theory recovers singular cohomology groups.

Let us first give the main ideas of the proof. Take two nondegenerate critical points $x$ and $y$ of a relative index 1 and a connecting orbit $C$. By the transversality, $\hat{S}=\{x, y, C\}$ is an isolated invariant set. Now choose a triple $\left(N_{2}, N_{1}, N_{0}\right)$ in such a way that the pairs $\left(N_{2}, N_{0}\right),\left(N_{2}, N_{1}\right)$ and $\left(N_{1}, N_{0}\right)$ are index pairs for the invariant sets $\hat{S},\{y\}$ and $\{x\}$, respectively (given a pair $\left(N_{2}, N_{0}\right)$ put $N_{1}=N_{2} \cap f^{-1}((-\infty, b])$ for an appropriate $\left.b\right)$.

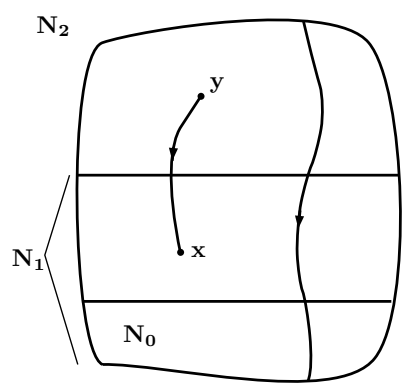

Figure 3

We have the following long exact sequence:

$$
\begin{aligned}
\cdots \rightarrow H_{E}^{k}\left(N_{2}, N_{0}\right) & \rightarrow H_{E}^{k}\left(N_{1}, N_{0}\right) \\
& \rightarrow H_{E}^{k+1}\left(N_{2}, N_{1}\right) \rightarrow H_{E}^{k+1}\left(N_{2}, N_{0}\right) \rightarrow \cdots .
\end{aligned}
$$

One can show that the cohomological Conley index for $S$ is trivial; i.e., all the groups $H_{E}^{n}\left(N_{2}, N_{0}\right)$ are trivial. Thus, for every $k$ we have an isomorphism

$$
H_{E}^{k+1}\left(N_{2}, N_{1}\right) \rightarrow H_{E}^{k}\left(N_{1}, N_{0}\right) .
$$


Abbondandolo computed (see [Abb97, Proposition 14.6]) the cohomological Conley index for a nondegenerate critical point:

$$
\operatorname{ch}^{k}(\{x\})= \begin{cases}\mathbb{Z}_{2} & \text { for } k=\operatorname{ind}_{E} x, \\ 0 & \text { otherwise. }\end{cases}
$$

The only nontrivial morphism in the exact sequence (4.1) is the isomorphism between $H_{E}^{k+1}\left(N_{2}, N_{1}\right)$ and $H_{E}^{k}\left(N_{1}, N_{0}\right)$ where $k=\operatorname{ind}_{E} x$.

By the compactness of $S$ and the transversality condition, we have finitely many orbits $C_{1}, C_{2}, \ldots, C_{m}$ connecting $y$ and $x$. Take

$$
\hat{S}=\left\{x, y, C_{1}, \ldots, C_{m}\right\} .
$$

By the additivity (see [McC, p. 201]), the Conley connection matrix is a sum of isomorphisms from $\mathbb{Z}_{2}$ to itself so it is an algebraic count modulo 2 . This is exactly the Floer boundary operator. Since $\operatorname{ch}^{*}(\{x\}) \simeq \mathbb{Z}_{2}$, one can think of $\operatorname{ch}^{*}(\{x\})$ as a generator of the Floer chain group $C_{\operatorname{ind}_{E} x}$.

Here are some technical details of the above construction. We would like to prove that the Conley index of $\hat{S}=\{x, y, C\}$ is trivial. Let us examine a special case. Suppose $C^{\prime}$ is contained in one-dimensional subspace $E_{1}$ and $x^{\prime}=(-1,0) \in E_{1} \oplus E_{1}^{\perp}, y^{\prime}=(1,0), C^{\prime}=[-1,1] \times\{0\}$.

Lemma 4.1. The Conley index of $\hat{S}$ is trivial.

Proof. We follow an approach of McCord [McC]; i.e., we use a series of continuations. Choose a small isolating neighborhood $N$ of $S^{\prime}$. First continue the vector field $F(x, y)=\left(F_{x}(x, y), F_{y}(x, y)\right)$ to

$$
\begin{aligned}
F_{1}(x, y) & =\left(F_{x}(x, 0)+D_{y} F_{x}(x, 0) y, F_{y}(x, 0)+D_{y} F(x, 0) y\right) \\
& =\left(F_{x}(x, 0)+D_{y} F_{x}(x, 0) y, D_{y} F(x, 0) y\right)
\end{aligned}
$$

and then to

$$
F_{2}(x, y)=\left(F_{x}(x, 0), D_{y} F(x, 0) y\right) .
$$

Now put $a(x)=F_{x}(x, 0), M=\max _{x \in[0,1]} a(x)$ and continue $F_{2}(x, y)$ to

$$
F_{3}(x, y)=\left(F_{x}(x, 0)-M-1, D_{y} F(x, 0) y\right) .
$$

Notice that $\operatorname{inv}\left(F_{3}, S\right)=\emptyset$ and thus the Conley index is trivial.

Now we would like to find an $E$-homotopy which reduces a general case to the above one (compare $[\mathrm{G}$, Section $\mathrm{C}]$ ).

Let $M$ be a compact $C^{1}$ submanifold of a Hilbert space $E$.

Lemma 4.2. There exists a finite-dimensional subspace $T$ of $E$ such that the orthogonal projection $P_{T}$ onto $T$ maps $M$ diffeomorphically onto $P_{T}(M)$.

Proof. For every $x \in M$ there is an open neighborhood $U_{x}$ such that $U_{x}$ is diffeomorphic to the open neighborhood of 0 in $T_{x} M$ via the exponential map. Choose a finite subcover $U_{x_{1}}, U_{x_{2}}, \ldots, U_{x_{k}}$ and put

$$
T^{\prime}=\operatorname{span}\left\{T_{x_{i}} M: i=1, \ldots, k\right\} .
$$


The orthogonal projection $P_{T^{\prime} \mid M}: M \rightarrow T^{\prime}$ is an imbedding. Thus, for a given $x \in M$, there is only a finite number of points $y_{1}, y_{2}, \ldots, y_{p}$ such that $P_{T^{\prime}} x=$ $P_{T^{\prime}} y_{i}$. Define $T_{x}^{\prime}$ as the space spanned by $T^{\prime}$ and $y_{1}-x, y_{2}-x, \ldots, y_{p}-x$ and let $P_{x}$ be the orthogonal projection onto $T_{x}^{\prime}$. It is easy to see that there is an open neighborhood $V_{x}$ of $x$ such that $y \in V_{x}, z \in M$ and $P_{x} z=P_{x} y$ imply $z=y$. Again, choose a finite cover $V_{x_{1}}, V_{x_{2}}, \ldots, V_{x_{q}}$ and put

$$
T=\operatorname{span}\left\{T_{i}^{\prime}: i=1, \ldots, q\right\}
$$

Lemma 4.3. There exist an $E$-homotopy and a finite-dimensional subspace $T_{1}$ such that

(1) $\Psi(\cdot, 0)=\mathrm{Id}$,

(2) $\Psi(M, 1)$ is contained in $T_{1}$.

Proof. By Lemma 4.2 we can find a finite-dimensional space such that $P_{T}$ : $M \rightarrow T$ is an injection. For $x \in M$ define $\phi_{x}: M \rightarrow E$ by

$$
\phi_{x}(y)=P_{T}(y-x)+x .
$$

Then $\phi_{x}$ is an imbedding and $\phi_{x}(x)=x$. Let $U_{x_{1}}, \ldots, U_{x_{k}}$ be a cover of $M$ and let $\left\{\nu_{i}\right\}$ be a subordinated partition of unity. Define $\phi: M \rightarrow E$ by

$$
\phi(x)=\sum \nu_{i}(x) \phi_{x_{i}}(x) .
$$

Take $T_{1}$ to be the space spanned by $T$ and $x_{1}, \ldots, x_{k}$. Then $\phi(M) \subset T_{1}$.

Define $\eta_{0}: P_{T}(M) \rightarrow T^{\perp}$ by

$$
\eta\left(P_{T} x\right)=x-\phi(x) .
$$

Since $P_{T}(M)$ is a $C^{1}$ submanifold of $T$, we can extend $\eta$ to a $C^{1}$ map on $T$. Define $\Psi(x, y)=x-t \eta\left(P_{T} x\right)$.

Two critical points together with an orbit between them is a compact submanifold of $E$. Thus we can apply the above lemma to $M=\hat{S}$. Suppose that $\Phi(\hat{S}, 1)$ is contained in a finite-dimensional space $T_{1}$. Choose a onedimensional subspace $E_{1} \subset T_{1}$ and a diffeomorphism $h$ of $T_{1}$ which takes $\hat{S}$ onto $(-1,1) \subset E_{1}$. Extend $h$ to $E$ by the identity on $T^{\perp}$. This reduces a general case to the one in Lemma 4.1 .

\section{Further directions}

Some of the Floer theories come with additional symmetry. One expects an analogous theorem to the main theorem of this paper for the equivariant Morse cohomology and the equivariant Conley index in a Hilbert space.

For an $S^{1}$-action there is the conjecture that the Monopole-Floer cohomology and the Seiberg-Witten-Floer cohomology are isomorphic (that is, $\left.\mathrm{HM}^{*}(Y) \simeq \mathrm{HSW}^{*}(Y)\right)$. However, this should be treated more carefully since one cannot assume the existence of the (local) flow in a Hilbert space. 
Another direction, that one would like to investigate, is the case of Hilbert (Banach) manifolds. Let us just recall that recently intensively explored Lagrangian intersection Floer theory (see [FOOO]) is a Floer theory on a Banach manifold.

\section{Acknowledgement}

I am indebted to K. Gęba and M. Izydorek, who provided valuable comments on an earlier version of this paper. I would also like to thank J. Maksymiuk for pointing out results in $[\mathrm{Ch}]$ and the referee for a number of helpful suggestions which improved the presentation paper.

\section{References}

[Abb97] A. Abbondandolo, A new cohomology for the Morse theory of strongly indefinite functionals on Hilbert spaces. Topol. Methods Nonlinear Anal. 9 (1997), 325-382.

[AGP] M. Aguilar, S. Gitler and C. Prieto, Algebraic Topology from a Homotopical Viewpoint. Springer-Verlag, New York, 2002.

[C-J] M. C. Crabb and J. Jaworowski, Aspects of the Borsuk-Ulam theorem. J. Fixed Point Theory Appl. 13 (2013), 459-488.

[Ch] K.-C. Chang, Methods in Nonlinear Analysis. Springer Monographs in Mathematics, Springer-Verlag, Berlin, 2005.

[FOOO] K. Fukaya, Y.-G. Oh, H. Ohta and K. Ono, Lagrangian Intersection Floer Theory: Anomaly and Obstruction. Part I. AMS/IP Stud. Adv. Math. 46, American Mathematical Society, Providence, RI, International Press, Somerville, MA, 2009.

[G] K. Gęba, Fredholm $\sigma$-proper maps of Banach spaces. Fund. Math. 64 (1969), 341-373.

[G-G] K. Gęba and A. Granas, Infinite dimensional cohomology theories. J. Math. Pures Appl. (9) 52 (1973), 145-270.

[GIP] K. Gęba, M. Izydorek and A. Pruszko, The Conley index in Hilbert spaces and its applications. Studia Math. 134 (1999), 217-233.

[G-D] A. Granas and J. Dugundji, Fixed Point Theory. Springer Monographs in Mathematics, Springer-Verlag, New York, 2003.

[Izy] M. Izydorek, A cohomological Conley index in Hilbert spaces and applications to strongly indefinite problems. J. Differential Equations 170 (2001), $22-50$.

[K-Sz] W. Kryszewski and A. Szulkin, An infinite-dimensional Morse theory with applications. Trans. Amer. Math. Soc. 349 (1997), 3181-3234.

[Man] C. Manolescu, Seiberg-Witten-Floer stable homotopy type of three-manifolds with $b_{1}=0$. Geom. Topol. 7 (2003), 889-932.

[McC] C. McCord, The connection map for attractor-repeller pairs. Trans. Amer. Math. Soc. 307 (1988), 195-203.

[Sal] D. Salamon, Morse theory, the Conley index and Floer homology. Bull. Lond. Math. Soc. 22 (1990), 113-140. 
[Smol] J. Smoller, Shock Waves and Reaction-Diffusion Equations. 2nd ed., Grundlehren Math. Wiss. 258. Springer-Verlag, New York, 1994.

[Sz] A. Szulkin, Cohomology and Morse theory for strongly indefinite functionals. Math. Z. 209 (1992), 375-418.

[Tau] C. F. Taubes, The Seiberg-Witten equations and the Weinstein conjecture. II. More closed integral curves of the Reeb vector field. Geom. Topol. 13 (2009), 1337-1417.

Maciej Starostka

Mathematical Institute

Polish Academy of Sciences

ul. Sniadeckich 8

00-956 Warsaw

Poland

and

Faculty of Technical Physics and Applied Mathematics

Gdansk University of Technology

Narutowicza 11/12

80-952 Gdansk

Poland

e-mail: maciejstarostka@gmail.com

Open Access This article is distributed under the terms of the Creative Commons Attribution License which permits any use, distribution, and reproduction in any medium, provided the original author(s) and the source are credited. 